\title{
openheart Cardiac magnetic resonance systematically overestimates mitral regurgitations by the indirect method
}

\author{
Litten Bertelsen (D , , Niels Vejlstrup, ${ }^{1}$ Laura Andreasen, ${ }^{1,2}$ Morten Salling Olesen, ${ }^{1,2}$ \\ Jesper Hastrup Svendsen ${ }^{1,3}$
}

\begin{abstract}
- Additional material is published online only. To view please visit the journal online (http://dx.doi.org/10.1136/ openhrt-2020-001323)
\end{abstract}

To cite: Bertelsen L, Vejlstrup N, Andreasen L, et al. Cardiac magnetic resonance systematically overestimates mitral regurgitations by the indirect method. Open Heart 2020;7:e001323. doi:10.1136/ openhrt-2020-001323

Received 23 April 2020 Revised 3 June 2020 Accepted 9 June 2020

\section{Check for updates}

(C) Author(s) (or their employer(s)) 2020. Re-use permitted under CC BY-NC. No commercial re-use. See rights and permissions. Published by BMJ.

${ }^{1}$ Department of Cardiology, Centre for Cardiac, Vascular, Pulmonary and Infectious Diseases, Rigshospitalet, Copenhagen University Hospital, Copenhagen, Denmark ${ }^{2}$ Department of Biomedical Sciences, University of Copenhagen, Copenhagen, Denmark

${ }^{3}$ Department of Clinical Medicine, University of Copenhagen, Copenhagen, Denmark

Correspondence to Dr Litten Bertelsen; littenbertelsen@gmail.com

\section{ABSTRACT}

Objective Cardiac MRI is quickly emerging as the gold standard for assessment of mitral regurgitation, most commonly with the indirect method subtracting forward flow in aorta from volumetric segmentation of the left ventricle. We aimed to investigate how aortic flow measurements with increasing distance from the aortic valve affect calculated mitral regurgitations and whether measurements were influenced by breath-hold regimen. Methods Free-breathing and breath-hold phase contrast flows were measured in aorta at valve level, sinotubular (ST) junction, mid-ascending aorta and in the pulmonary trunk. Flow measurements were pairwise compared, and subsequently, after exclusion of patients with visible mitral and tricuspid regurgitations for left-sided and right-sided comparisons, respectively, flow-measured stroke volumes were compared with ventricular volumetric segmentations. Results Thirty-nine participants without arrhythmias or structural abnormalities of the large vessels were included. Stroke volumes measured with free-breathing and breath-hold flow decreased equally with increasing distance to the aortic valves (breath-hold flow: aortic valve $105.6 \pm 20.8 \mathrm{~mL}$, ST junction $101.5 \pm 20.7 \mathrm{~mL}$, mid-ascending aorta $98.1 \pm 21.5 \mathrm{~mL}$ ). After exclusion of atrioventricular regurgitations, stroke volumes determined by volumetric measurements were higher compared with values determined by flow measurements, corresponding to 'false' atrioventricular regurgitations of $8.0 \% \pm 5.8 \%$ with flow measured at valve level, $11.6 \% \pm 5.2 \%$ at the ST junction and $15.3 \% \pm 5.0 \%$ at the mid-ascending aorta. Conclusions Stroke volumes determined by flow decrease throughout the proximal aorta and are systematically lower than volumetrically measured stroke volumes. The indirect method systematically overestimates mitral regurgitations, especially with increasing distance from the aortic valves.

\section{INTRODUCTION}

Cardiovascular magnetic resonance imaging (CMR) is the gold standard for measuring volumes in the heart and is widely used for this purpose in research and clinical practice. Mitral regurgitations can be assessed by CMR with the indirect method by subtracting the forward phase contrast (PG) flow in aorta from the detailed volumetric segmentation of

\section{Key questions}

What is already known about this subject?

- The indirect method for quantification of mitral regurgitations is reproducible and superior to echocardiographic methods. Literature suggests that stroke volumes measured with phase contrast flow in aorta decrease with increasing distance from the aortic valve, but there is no consensus on where to measure flow in aorta and how choice of level impacts calculated mitral regurgitations by the indirect method.

What does this study add?

- In participants with no structural abnormalities of the large vessels, stroke volumes measured with flow in aorta decreases with increasing distance from the aortic valves and stroke volumes are systematically lower when compared with volumetric measurements irrespective of breath-hold regimen. This results in an overestimation of calculated mitral regurgitations when using the indirect method ranging from $8 \%$ at the aortic valve level, $12 \%$ at the sinotubular junction and $15 \%$ in the mid-ascending aorta.

How might this impact on clinical practice?

- A clear indication of the level of measurement should always be reported for clinicians to assess how measurement method could possibly affect results. Choice of distal level of measurement may result in overestimation of mitral regurgitations.

the left ventricle. ${ }^{1}$ A recent review ${ }^{2}$ highlights CMR as superior to 2D and 3D echocardiography in quantification of mitral regurgitations and advocates the use of CMR in future studies and clinical assessments. Despite its many advantages, CMR is also subject to method-specific errors and it is essential that these limitations are known and taken into account when reporting CMR-derived measurements. $^{3}$

CMR studies have shown that stroke volume (SV) determined in the ascending aorta with 2D PC flow in healthy controls decrease the more distally in the ascending 
aorta the measurement is performed, ${ }^{45}$ and the phenomenon is even more pronounced under pathological conditions. ${ }^{5-8}$ While a recent consensus statement recommends measuring forward aortic volume at the level of the sinotubular (ST) junction, ${ }^{9}$ different measurement locations in the aorta are regularly used (table 1). In some studies, measurements from different locations are used indiscriminately, ${ }^{10}$ and some studies do not report the location of flow measurement. ${ }^{11-13}$

Furthermore, studies have found a systematic difference, with volumetrically segmented SV being several millilitres greater than SV based on flow measurements. $^{81415}$ Consequently, this may result in systematic overestimation of calculated atrioventricular regurgitations. Theoretically, this could be caused by different breath-hold regimens between volumetric and flow measurements. ${ }^{16}$ It has been suggested that, if performed correctly, free-breathing and breath-hold PC flow can be used interchangeably ${ }^{717}$ while others disagree. ${ }^{4}$

The aim of this study was to quantify the impact of changing image planes between the most frequently used locations in aortic flow measurements. Second is to evaluate the impact of breath-hold regimen as well as evaluate the corresponding 'false' regurgitant volume with the indirect method. To provide a best-case scenario, the study was performed in subjects with no abnormalities of the large vessels.

\section{METHODS}

Participants were recruited from a clinical study including patients with known paroxysmal atrial fibrillation and a healthy control group from October to December 2017. All included participants had a full CMR and no previously known structural heart disease. Participants were excluded if they could not comply with instructions, experienced arrythmia during the scan, if CMR revealed pathological conditions of valves or vessels of interest, or if any flow measurements were of unacceptable quality.

All participants were at least 18 years of age and signed informed written consent prior to scanning. Patient or public involvement was not considered appropriate.

\section{Image acquisition}

All scans were performed on a $1.5 \mathrm{~T}$ Siemens Aera (Erlangen, Germany) and corresponded to normal clinical scans.

Long-axis cine images (two-chamber, three-chamber and four-chamber) were acquired initially. Volumetric measurements were performed with SSFP cine sequences

\section{Table 1 Overview of studies on assessment of mitral regurgitation with CMR}

\begin{tabular}{|c|c|c|c|}
\hline First author and reference & Year & $\mathbf{n}$ & Reported level of measurement \\
\hline Hundley et al ${ }^{1}$ & 1995 & 17 & 2 to $4 \mathrm{~cm}$ above the aortic valve \\
\hline Kizilbash et al & 1998 & 22 & Ascending aorta, well above the aortic valve and coronary ostia \\
\hline Gelfand et al & 2006 & 83 & The axial plane at the level of the bifurcation of the pulmonary artery \\
\hline Buck et al & 2008 & 73 & Orthogonal to the mid-ascending aorta \\
\hline Uretsky et $\mathrm{al}^{12}$ & 2010 & 23 & Not reported \\
\hline Myerson et $a l^{10}$ & 2010 & 55 & Just above the aortic valve \\
\hline Shanks et al & 2010 & 30 & $\begin{array}{l}2 \text { to } 5 \mathrm{~cm} \text { above the aortic valve, where the aorta was nearly parallel to the caudal } \\
\text { cranial axis of the patient, in the axial orientation }\end{array}$ \\
\hline Hamada et al & 2012 & 43 & $10 \mathrm{~mm}$ above the tip of the aortic valve \\
\hline Cawley et al & 2013 & 26 & Aortic valve \\
\hline Van De Heyning et al & 2013 & 38 & Ascending aorta \\
\hline Thavendiranathan et al & 2013 & 30 & 1 to $2 \mathrm{~cm}$ above the aortic valve \\
\hline Choi et al & 2014 & 52 & 2 to $3 \mathrm{~cm}$ above the aortic valve \\
\hline Uretsky et al ${ }^{11}$ & 2015 & 103 & Not reported \\
\hline Sachdev et $a l^{13}$ & 2017 & 58 & Not reported \\
\hline Lopez-Mattei et al & 2016 & 70 & Sinotubular junction \\
\hline Aplin et $a l^{22}$ & 2016 & 72 & Sinotubular junction \\
\hline Myerson et $\mathrm{al}^{23}$ & 2016 & 109 & Just above the aortic valve at end diastole or at the sinotubular junction \\
\hline Harris et al & 2017 & 22 & Across the aortic valve \\
\hline Polte et al & 2017 & 40 & Sinotubular junction \\
\hline Heo et al & 2017 & 37 & 2 to $3 \mathrm{~cm}$ above the aortic valve \\
\hline Penicka et $a l^{24}$ & 2018 & 258 & 2 to $3 \mathrm{~cm}$ above the aortic valve \\
\hline Chew et al ${ }^{30}$ & 2019 & 109 & At the aortic sinotubular junction or just above the valve prosthesis post-replacement \\
\hline
\end{tabular}

CMR, cardiac magnetic resonance imaging. 


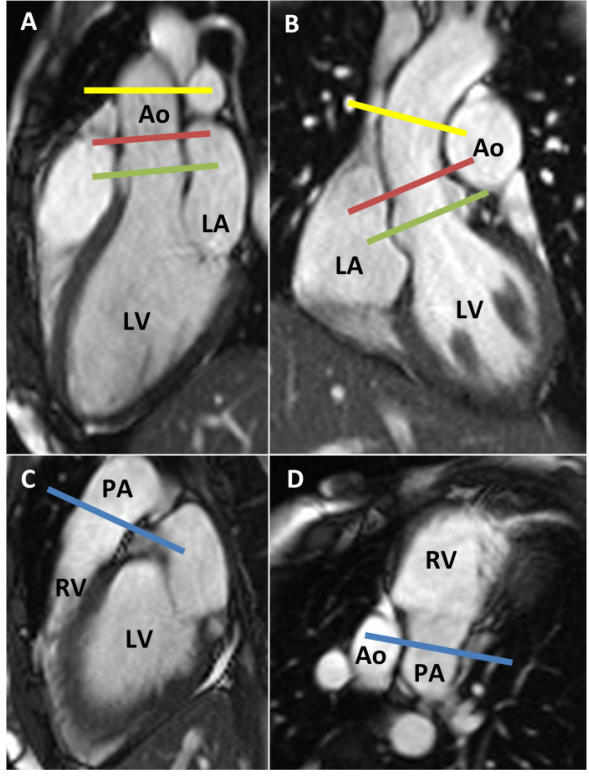

Figure 1 Illustration of included flow measurements. (A, B) Three-chamber and orthogonal cine images showing levels of flow measurements in aorta at valve level (green line), sinotubular junction (red line) and mid-ascending aorta (yellow line). (C, D) Right ventricular outflow tract and pulmonary trunk and orthogonal cine images showing level of flow measurement in the pulmonary artery (blue line). Ao, aorta; LA, left atrium; LV, left ventricle; PA, pulmonary artery/ trunk; RV, right ventricle.

(typical imaging parameters: $8 \mathrm{~mm}$ slice thickness; $2 \mathrm{~mm}$ gap; pixel size $1.9 \times 1.9 \mathrm{~mm}$; 25 phases; TR/TE 2.6/1.1 ms) during end-expiratory breath-holds. An axial stack ranging from basis to the aortic arch and a short-axis stack covering the entire left ventricle were obtained.

Flow (through plane phase contrast) sequences corresponding to normal clinical flow sequences were performed. A free-breathing (FB) and a breathhold $(\mathrm{BH})$ sequence were performed at each plane as depicted in figure 1: in the pulmonary trunk (PA) and at three levels in the aorta. For measurements at aortic valve level (Va), the imaging plane was placed at the tip of the open aortic valve leaflets in systole always proximal to the coronary arteries. At the ST junction, the imaging slice was placed just distal to the aortic sinus. At the midascending aorta, the slice in the axial stack displaying the pulmonary bifurcation was copied without corrections to the flow sequence as described in the literature. ${ }^{18}$ For positioning of all flow planes (except the mid-ascending aorta) perpendicularly to the vessels, two orthogonal images were obtained. Imaging parameters for the two flow sequences are reported in table 2. To minimise phase offset errors, vessels were placed along the z-axis as close to the isocentre as possible. Velocity encoding was set to $200 \mathrm{~cm} / \mathrm{s}$.

\section{Image analyses}

All images were analysed in CVI $^{42}$ V.5.6.6 (Circle Cardiovascular Imaging). On short-axis cine images, left ventricular (LV) end-diastolic and end-systolic phases
Table 2 Acquisition parameters for phase contrast flow sequences

\begin{tabular}{lll}
\hline & Breath-hold & Free-breathing \\
\hline TR/TE $(\mathrm{ms})$ & $4.64 / 2.47$ & $5.09 / 2.8$ \\
\hline Flip angle $\left(^{\circ}\right)$ & 20 & 20 \\
\hline Bandwidth (Hz/pixel) & 450 & 455 \\
\hline Slice thickness $(\mathrm{mm})$ & 6 & 5 \\
\hline Matrix size (voxels) & $(126-192) \times(126-256)$ & $(168-256) \times(176-256)$ \\
\hline Pixel size $(\mathrm{mm})$ & $1.77 \times 1.77$ & $1.33 \times 1.33$ \\
\hline Frames/cycle & 30 & 50 \\
\hline $\begin{array}{l}\text { Typical acquisition } \\
\text { time }(\mathrm{s})\end{array}$ & $13-17$ & $150-180$ \\
Averages & 1 & 3 \\
\hline
\end{tabular}

TE, echo time; TR, repetition time.

were traced manually at the endocardial and epicardial border. LV outflow tract was included in the blood pool; papillary muscles were excluded, using windowing for the endocardial border. Epicardium was delineated in both phases to compare left myocardial mass in end-diastole and end-systole. The right ventricle was traced manually on axial cine images in end-diastolic and end-systolic phases and trabeculation was included in the blood pool. Right ventricular outflow tract was included in the blood pool. Cross-sectional images of valves were carefully referenced to determine distinction between ventricular blood pool and atrial and vascular blood pool.

Flow sequences were measured semi-automated with manual correction, carefully tracing the outer vessel wall. Images and flow curves were assessed for signs of arrhythmia during scanning and aliasing. As we wanted the setting to be as similar to a clinical workflow as possible, we did not perform phantom corrections. Background correction was performed on all uniform muscle tissue in the magnitude image. ${ }^{15} 19$ Forward flow, representing $\mathrm{SV}$, was registered for all images (pulmonary artery, aorta at valve level, ST junction and mid-ascending aorta with breath-hold and free-breathing sequences, respectively). Heart rate for each sequence was registered. Peak velocity for each flow measurement was registered in $2 \times 2$ pixels to avoid errors on pixel level. ${ }^{20} \mathrm{Q}_{\mathrm{p}} / \mathrm{Q}_{\mathrm{s}}$ ratios were calculated as pulmonary SV measured with PC flow divided by systemic SV measured with PC flow at different levels in the aorta.

The axial cine stack and two-chamber, three-chamber and four-chamber cine images were visually inspected for mitral and tricuspid regurgitations. The flow measurements were not taken into consideration when assessing valvular regurgitation.

\section{Statistics}

All data are reported as means and SD unless stated otherwise. Paired t-tests were performed to compare flow measurements. $\mathrm{P}$ values less than 0.05 were considered statistically significant. 
First, to assess the effect of breathing regimen, flow assessments with $\mathrm{FB}$ and $\mathrm{BH}$ sequences were compared for each imaging level with paired t-tests and BlandAltman plots were constructed..$^{21}$ Due to the flow in the coronary arteries, flow comparisons were limited to flow in aorta at valve level versus the pulmonary artery and in aorta at ST junction versus mid-ascending aorta. BlandAltman plots were visually inspected for trumpet shapes as a sign of increasing effect with increasing SV.

Subsequently, for assessment of a possible bias when calculating mitral regurgitations with the indirect method, differences between flow and volumetric measurements were compared with paired t-tests and Bland-Altman plots. In this second analysis, participants with visible mitral or tricuspid regurgitations were excluded from left-sided and right-sided measurements, respectively. The resulting 'false' regurgitant volume was calculated for each measurement.

\section{RESULTS}

Initially, 50 participants were included. Three participants were excluded due to frequent premature ventricular contractions. Three participants were excluded due to inability to comply with BH instructions. Two participants were excluded due to bicuspid aortic valves (not previously known). Three participants were excluded due to a single flow measurement of unacceptable quality (two FB valve flows due to inability to make a precise delineation of the valve because of too much blurring and one BH pulmonary flow due to wrap over the artery, not noticed during the scan). Consequently, 39 participants with no abnormalities of the large vessels and complete flow measurements were included in the analyses. Nine participants had visible mitral regurgitation. Thirteen had visible tricuspid regurgitation. Baseline characteristics of participants are reported in table 3 .

\section{BH regimens}

There were no statistical differences between $\mathrm{FB}$ and $\mathrm{BH}$ flows, except at valve level (table 4). As summarised in online supplementary table S1, background correction changed $\mathrm{FB}$ more than $\mathrm{BH}$ sequences, hence $\mathrm{FB}$ and BH flow measurements before background correction were statistically different. Bland-Altman plots of FB flow compared with BH flow showed no increased difference with increasing SV (online supplementary figure S1). The limits of agreement were $\pm 14.3 \mathrm{~mL}$ for the pulmonary artery while similar for all aorta flows (valve $\pm 9.9 \mathrm{~mL}$, ST junction $\pm 10.4 \mathrm{~mL}$ and mid-ascending $\pm 9.9 \mathrm{~mL}$ ), and all showed none to very small bias from zero.

Comparisons of peak velocities, heart rates and artefacts between $\mathrm{FB}$ and $\mathrm{BH}$ sequences are reported in online supplementary material.

\section{Flow signal in the aortic root}

Table 4 summarises the SV measured at the three different levels in the aortic root and in the main pulmonary artery. A decrease in aortic flow volume was seen with
Table 3 Baseline characteristics of included patients

\begin{tabular}{|c|c|}
\hline $\mathbf{N}$ & 39 \\
\hline Age, years (IQR) & $42.5(38.6-45.1)$ \\
\hline Height, cm (SD) & $182.2(8.6)$ \\
\hline Weight, kg (SD) & $86.1(11.4)$ \\
\hline Male sex, n (\%) & $32(82)$ \\
\hline Hypertension, n (\%) & 3 (2 without treatment) (8) \\
\hline Diabetes mellitus, n (\%) & $0(0)$ \\
\hline Ischaemic heart disease, $\mathrm{n}(\%)$ & $0(0)$ \\
\hline Paroxysmal atrial fibrillation, $\mathrm{n}(\%)$ & $26(67)$ \\
\hline LV EDV, mL/m² (SD) & $92.5(14.7)$ \\
\hline LV ESV, mL/m² (SD) & $37.7(7.8)$ \\
\hline LV SV, mL/m² (SD) & $54.9(9.2)$ \\
\hline LV EF (\%) & $59.4(5.0)$ \\
\hline $\begin{array}{l}\text { LV myocardial mass (diastole), g/ } \\
\mathrm{m}^{2}(\mathrm{SD})\end{array}$ & $62.4(11.0)$ \\
\hline RV EDV, mL/m² (SD) & $96.9(17.2)$ \\
\hline RV ESV, $\mathrm{mL} / \mathrm{m}^{2}$ (SD) & $43.1(9.1)$ \\
\hline RV SV, mL/m² (SD) & $53.7(9.7)$ \\
\hline RV EF (\%) & $55.6(4.4)$ \\
\hline
\end{tabular}

Values are shown as mean (SD) or median (IQR).

EDV, end-diastolic volume; EF, ejection fraction; ESV, end-systolic volume; LV, left ventricle; RV, right ventricle; SV, stroke volume.

increasing distance to the valve. Figure 2 depicts how this was directly evident in the majority of the participants. A decrease of $5 \%$ would be expected between aortic valve and ST junction, due to coronary flow, but the additional decrease between ST junction and mid-ascending aorta was also statistically significant. The corresponding $Q_{p} /$ $Q$ ratios also increased with increasing distance from the valves (table 4).

\section{Bias between flow and volumetric measurements}

Figure 3 shows Bland-Altman plots for FB and BH flow measurements compared with corresponding volumetric measurements. In these comparisons, participants with visible mitral regurgitations (for left-sided comparisons) and tricuspid regurgitations (for right-sided comparison) have been excluded and therefore the volumetric measurements should theoretically be equal to SV measured with flow, though $5 \%$ lower when measured in the aorta distal to the coronary arteries. Nevertheless, we found a bias between volumetric measurements and $\mathrm{BH}$ flow $\mathrm{SV}$ increasing from $10.6 \mathrm{~mL}$ on valve level to $14.0 \mathrm{~mL}$ on ST junction and further to $17.7 \mathrm{~mL}$ in mid-ascending aorta. On the right side, a mean difference of $4.8 \mathrm{~mL}$ was found. Comparisons with FB showed similar results for ST junction $(15.3 \mathrm{~mL})$ and mid-ascending aorta $(17.8 \mathrm{~mL})$ but a smaller bias at valve level $(6.4 \mathrm{~mL})$ (online supplementary table S2).

In clinical assessment of mitral or tricuspid regurgitations, the found bias would, employing the indirect method, result in a 'false' regurgitant volume as presented 
Table 4 Mean stroke volumes measured in aorta and in the main pulmonary artery and corresponding $Q_{p} / Q_{s}$ ratios

\begin{tabular}{|c|c|c|c|}
\hline $\mathrm{n}=39$ & FB & $\mathrm{BH}$ & $\begin{array}{l}\text { P value } \\
\text { FB vs BH }\end{array}$ \\
\hline \multicolumn{4}{|l|}{ Valve level } \\
\hline Stroke volume, mL (SD) & $107.5(20.2)$ & $104.1(19.6)$ & \multirow[t]{2}{*}{$<0.001$} \\
\hline$Q_{p} / Q_{s}$, mean (range) & $0.98(0.78-1.10)$ & $1.02(0.85-1.21)$ & \\
\hline \multicolumn{4}{|l|}{ ST-junction level } \\
\hline Stroke volume, mL (SD) & $99.1(20.2)$ & $99.9(20.2)$ & \multirow[t]{2}{*}{0.3} \\
\hline$Q_{p} / Q_{s}$, mean (range) & $1.06(0.89-1.29)$ & $1.07(0.96-1.23)$ & \\
\hline \multicolumn{4}{|l|}{ Mid-ascending aorta } \\
\hline Stroke volume, mL (SD) & $96.2(20.8)$ & $96.2(20.6)$ & \multirow[t]{2}{*}{1.0} \\
\hline$Q_{p} / Q_{s}$, mean (range) & $1.10(0.94-1.21)$ & $1.11(0.91-1.29)$ & \\
\hline \multicolumn{4}{|l|}{ Pulmonary artery } \\
\hline Stroke volume, mL (SD) & $104.5(21.1)$ & $106.0(20.5)$ & 0.2 \\
\hline \multicolumn{4}{|l|}{$P$ values for flow vs flow } \\
\hline Valve vs pulmonary & 0.02 & 0.1 & \\
\hline ST junction vs mid-ascending & 0.01 & $<0.001$ & \\
\hline
\end{tabular}

$P$ values for paired t-tests between stroke volumes.

$Q_{p} / Q_{s}$ should be 1 before the coronary arteries (valve level) and 1.05 after the coronary arteries (ST junction and mid-ascending aorta).

$\mathrm{BH}$, breath-hold; FB, free-breathing; $\mathrm{Q}$, flow rate; $\mathrm{Q}_{\mathrm{p}} / \mathrm{Q}_{\mathrm{s}}$, pulmonary flow divided by systemic flow; $\mathrm{ST}$ junction, sinotubular junction.

in figure 4. This overestimated volume resulted in calculated regurgitation fractions in healthy young participants with no visible regurgitation of $8.0 \%$ (SD 5.8) for BH measurements at valve level, $11.6 \%$ (SD 5.2) at the ST junction, $15.3 \%$ (SD 5.0) at the mid-ascending aorta and $3.8 \%$ (SD 8.3 ) in the pulmonary artery.

\section{DISCUSSION}

This study demonstrates fundamental problems in assessment of mitral regurgitation using the most commonly applied CMR approach. Our study shows that in subjects
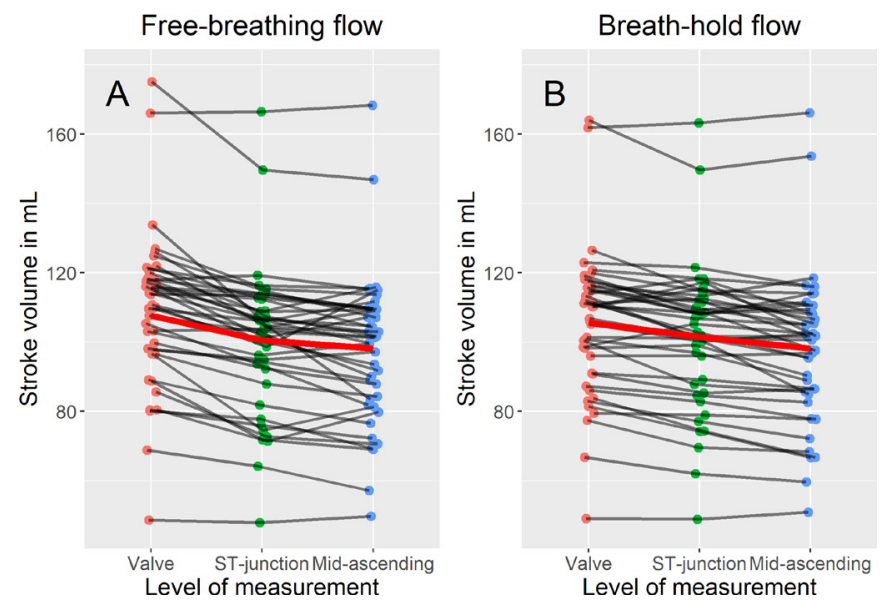

Figure 2 Individual flow measurements in aorta. Stroke volumes measured with phase contrast flow at indicated levels in the aortic root (valve, ST junction and midascending). (A) Free-breathing flow measurements. (B) Breath-hold flow measurements. Red line indicates mean values. Notice how measured stroke volumes decrease with increasing distance to the valve. ST, sinotubular. with no abnormalities of the large arteries, CMR 2D PC flow measurements decrease from the aortic valve throughout the aortic root and ascending aorta. Compared with volumetric measurements, flow measurements underestimate SV. This difference results in a calculated 'false' atrioventricular regurgitant volume when applying the most common method for assessing mitral regurgitations, the indirect method. Calculated mitral regurgitations increased from $8 \%$ at valve level to $15 \%$ in the mid-ascending aorta. The difference was not affected by different $\mathrm{BH}$ regimens.

As described in recent reviews, ${ }^{23}$ CMR quantification of mitral regurgitation is reproducible and has shown its superiority compared with other methods in large studies. ${ }^{11} 22-24$ Even though quantification of aortic flow is essential to the indirect CMR assessment of regurgitations, some of these studies use the different measurement planes equally or do not report the location of the aortic flow measurement (table 1). Hence, based on the results from the present study, these studies may have overestimated calculated mitral regurgitations by up to $15 \%$. Because the conditions in the present study represent a best-case scenario, the actual overestimation is possibly even larger.

\section{Mitral regurgitation assessments with the indirect method}

A different issue with the indirect quantification method is the combination of two different means of measurement, the volumetric segmentation and the forward flow measurement. First, the measurements are not performed simultaneously and a change in cardiac output during the scan, which is not uncommon due to initial patient nervousness associated with being in the scanner and 

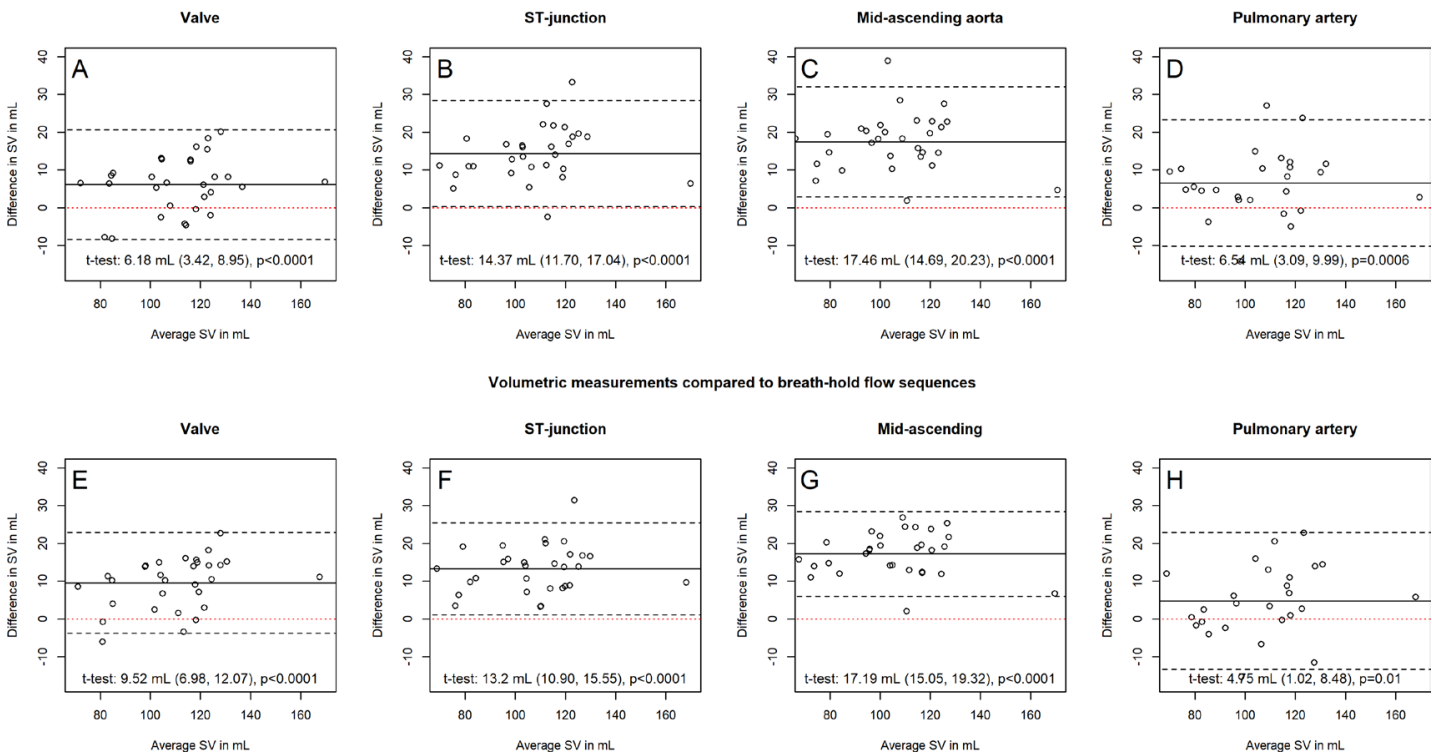

Figure 3 Bland-Altman plots volumetric vs flow measurements. The continuous line represents the mean and the dotted line represent the $95 \%$ limits of agreement for SV measured with FB and BH flow, respectively, at indicated place compared with corresponding volumetric measurement. Dotted red line indicates no bias. (A, E) Valve level. (B, F) ST junction. (C, G) Midascending aorta. (D, H) Pulmonary artery. Corresponding paired t-tests are indicated. In left-sided measurements, patients with mitral regurgitation have been excluded $(n=30)$. In right-sided measurements patients with tricuspid regurgitation have been excluded ( $n=26)$. BH, breath-hold; FB, free-breathing; ST, sinotubular; SV, stroke volume.

subsequent accommodation to the unfamiliar environment, often results in a fall in cardiac output. Second, reproducibility tests of PC flow have shown high consistency of mean values and when analyses are repeated on the same images or images are repeated on the same patient, the SV measured with PC flow vary, typically in the range of $\pm 10-15 \mathrm{~mL}^{4}{ }^{12} 25$ The volumetric measurements also provide high reproducibility in the range of approximately $\pm 10 \mathrm{~mL} .{ }^{26}$ Therefore, calculations of mitral regurgitations combining a volumetric and a flow measurement increase the variation and a true value should be expected within approximately $\pm 20-25 \mathrm{~mL}$. Hence, studies on many patients may provide high reproducibility and precision, but in clinical praxis we often struggle with variation in measurements and how to interpret the results for the individual patient. This issue is highlighted by Gulsin $e t a l^{27}$ stating that it is not uncommon that measured flow is higher at the ST junction compared with valve level even though the opposite would be expected due to the position of the coronary arteries between the two locations. This is also evident in figure 2 where flow SV of a few participants are not decreasing throughout the aortic root as it is for the majority. This may in part be due to the variation seen in flow measurements and of course be due to the fact that PC flow is very susceptible to artefacts and arrhythmias. ${ }^{28} 29$

To further complicate matters, in contrast to the presently found underestimation of forward flow in aorta when compared with volumetric assessment, the presence of aortic stenoses and hence high flow velocities often result in overestimation of forward flow in aorta. ${ }^{830}$ Thus, in this context, the indirect method may result in underestimation of possible mitral regurgitations, which is often coexisting with aortic stenosis. ${ }^{30}$

The indirect method furthermore relies on the volumetric assessment as reference. Despite being the gold standard, ${ }^{18}$ this method has several limitations. Results of volumetric measurements are highly dependent on uniform BHs, correct delineation and especially the basal slice will have a major contribution to the results, and it is thus important whether it is included in the blood pool or not, though the slice may be precisely in the mitral valve.

\section{Turbulence and complex flow}

A previous study has speculated that the systematic difference between flow and volumetric measurements could be caused by the different physiology during $\mathrm{BH}$ used for cine sequences versus $\mathrm{FB}$ used for flow sequences. ${ }^{8}$ The present study contradicts this hypothesis because $\mathrm{FB}$ and $\mathrm{BH}$ flow sequences produce similar results, suggesting that the observed differences between volumetric and 2D PC flow measurement may be an inherent problem with the 2D PC flow method. The flow pattern in the ascending aorta is complex, though under normal conditions approximately laminar until late systole where it forms a right-handed helix pattern as well as vortical flow (flow resolving around an axis) in the sinus of Valsalva. In patients with pathological conditions, the flow becomes more complex and forms turbulence, acceleration and a more pronounced vortical and helical flow pattern. ${ }^{31}$ As 2D PC flow is only measuring flow in one plane, it is prone to underestimation and complex flow patterns will result in lower volumes measured. Several studies have found clearly reduced 

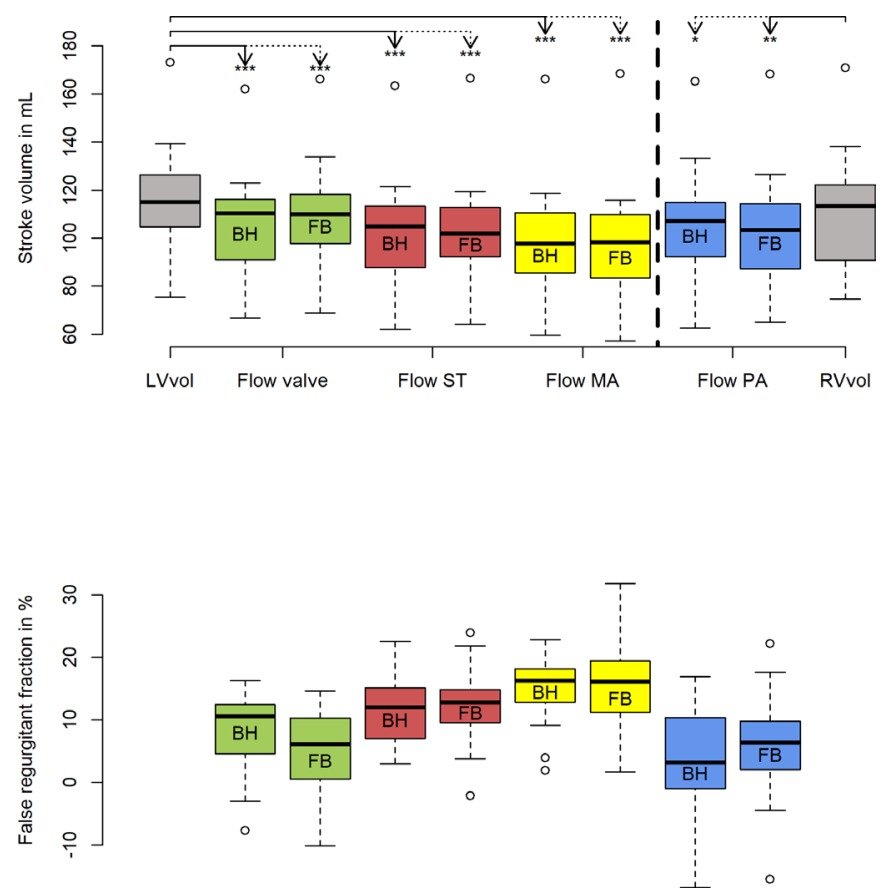

Figure 4 Measured stroke volumes and corresponding calculated 'false' regurgitant fraction. Mean stroke volumes measured volumetrically in left (LVvol) and right (RVvol) ventricle and with $\mathrm{FB}$ and $\mathrm{BH}$ flow at valve level (flow valve), sinotubular junction (flow ST), mid-ascending aorta (flow MA) and the pulmonary artery (flow PA). In left-sided measurements (LVvol, flow valve, flow ST and flow MA), patients with mitral regurgitation have been excluded $(n=30)$. In right-sided measurements (RVvol and flow $P A)$, patients with tricuspid regurgitation have been excluded $(n=26)$. Results are summarised in online supplementary table S2. Note that LV volumetric and flow at valve level should theoretically be similar, but that flow at the ST junctions is expected to be $5 \%$ lower because of the coronary arteries. A further fall at the mid-ascending aorta is not expected. The RV-volumetric and pulmonary flow should theoretically be similar as well. Below the x-axis are the corresponding calculated atrioventricular regurgitation fractions. Regurgitant volume is calculated by subtracting forward phase contrast flow in aorta at specified measurements level from the volumetric segmentation. ${ }^{\star} \mathrm{p}=0.01,{ }^{* *} \mathrm{p}<0.001,{ }^{\star * *} \mathrm{p}<0.0001$. $\mathrm{BH}$, breath-hold flow; FB, free-breathing flow; LV, left ventricle; $\mathrm{RV}$, right ventricle; $\mathrm{ST}$, sinotubular.

measured 2D PC aortic flow in patients with increased turbulence, ${ }^{568}$ while as demonstrated by Nordmeyer $e t a l$, this is not a problem with $4 \mathrm{D}$ flow in healthy volunteers. ${ }^{31}$ The found reduction in measured SV may very well be a result of increasing turbulence with increasing distance to the valve, even in healthy subjects. $4 \mathrm{D}$ flow furthermore allows direct quantification of regurgitant volumes employing a dynamic measurement plane by tracking the mitral annulus throughout the cardiac cycle. ${ }^{32}$ Studies suggest ${ }^{3133}$ that $4 \mathrm{D}$ flow measurements could possibly solve many of the issues addressed in the present paper, but besides requiring extensive training, 4D flow is currently not commercially available on all scanners.
In 2D PC imaging, it is very important that flow is perpendicular to the imaging slice. Valve images and ST-junction images were carefully planned orthogonally to aorta whereas the mid-ascending images were copied from the axial stack and hence could result in some angulation to the aorta. This would be expected to be within \pm 15 degrees that should be compensated for by the simultaneous increase in vessel area and increase in partial volume effects, ${ }^{29}{ }^{34}$ thereby not causing the lower $\mathrm{SV}$ measured at the mid-ascending aorta.

\section{Clinical flow measurements}

For a clinical workflow, the optimal flow assessment should have no phase offset error and should require very little post-processing work. Intuitively, the mid-ascending aorta seems the obvious choice. However, as indicated by the present study, in choosing such a distal image position in the aorta, one also introduces a significant systematic error. This was evident for $\mathrm{FB}$ as well as $\mathrm{BH}$ sequences. Essential to this point, our study provides a best-case scenario since the subjects in the present study were all healthy. Nevertheless, flow measurements systematically decrease in aorta and result in increasing overestimation of mitral regurgitation of up to $15 \%$. Structural abnormalities in the aortic valve or ascending aorta, which are often present in patients referred for CMR, may introduce even larger errors. ${ }^{56}$ It is unpredictable how large the loss of SV due to turbulence in each case will be and it can therefore not simply be corrected for by adding the approximated 'lost volume'.

When studying atrioventricular valve regurgitations using CMR, we therefore recommend that

- Multiple CMR techniques are used in order to get a detailed description of the condition and a reliable conclusion. ${ }^{9}$

- Flow is reported as a mean of at least two consecutive acquisitions due to the possible errors in flow measurements.

- Flow measurements in the mid-ascending aorta are avoided or used with caution.

- Background correction is applied and the effect on the results evaluated.

\section{Limitations}

All scans were performed on a Siemens Aera scanner, and results could vary on other magnetic resonance scanners.

$\mathrm{PC}$ sequence parameters for $\mathrm{FB}$ and $\mathrm{BH}$ were not identical (table 2) which may impact the comparison between these methods. The sequences used correspond to clinical scans, and the results are hence clinically applicable.

A limitation of the present study is the use of the volumetric assessment as reference. As described previously, this similarly applies to the indirect method.

We only performed flow in the pulmonary trunk at one level and cannot say whether flow signal changes throughout the pulmonary trunk. 


\section{CONCLUSION}

Mitral regurgitations are systematically overestimated by the indirect method combining CMR PC flow and volumetric segmentations. The overestimation ranged from $8.0 \%$ at valve level to $12 \%$ at the ST junction, and $15 \%$ at the mid-ascending aorta in participants with no abnormalities of the large vessels and no visible atrioventricular regurgitations. There were no differences between $\mathrm{BH}$ and $\mathrm{FB}$ sequences.

Contributors LB conceptualised and designed the study, performed data acquisition and measurements, performed statistical analyses and drafted the manuscript. LA contributed to study design, performed data acquisition and revised the manuscript. NV, MSO and JHS contributed to study design, participated in scientific discussions during the study and revised the manuscript. All authors read and approved the final version of the manuscript.

Funding The study is supported by the Innovation Fund Denmark (12-135225) and the Arvid Nilssons Fond (no grant number).

Competing interests JHS reports to be a member of Medtronic advisory boards and to have received speaker honoraria and research grants from Medtronic and Gilead not related to this work.

Patient consent for publication Not required.

Ethics approval The study was approved by the local ethics committee of the Capital Region of Denmark (protocol no. H-1-2011-044).

Provenance and peer review Not commissioned; externally peer reviewed.

Data availability statement Data are available on reasonable request.

Open access This is an open access article distributed in accordance with the Creative Commons Attribution Non Commercial (CC BY-NC 4.0) license, which permits others to distribute, remix, adapt, build upon this work non-commercially, and license their derivative works on different terms, provided the original work is properly cited, appropriate credit is given, any changes made indicated, and the use is non-commercial. See: http://creativecommons.org/licenses/by-nc/4.0/.

ORCID iD

Litten Bertelsen http://orcid.org/0000-0001-7852-6537

\section{REFERENCES}

1 Hundley WG, Li HF, Willard JE, et al. Magnetic resonance imaging assessment of the severity of mitral regurgitation. Comparison with invasive techniques. Circulation 1995;92:1151-8.

2 Uretsky S, Argulian E, Narula J, et al. Use of cardiac magnetic resonance imaging in assessing mitral regurgitation: current evidence. J Am Coll Cardiol 2018;71:547-63.

3 Krieger EV, Lee J, Branch KR, et al. Quantitation of mitral regurgitation with cardiac magnetic resonance imaging: a systematic review. Heart 2016;102:1864-70.

4 Bolen MA, Setser RM, Gabriel RS, et al. Effect of protocol choice on phase contrast cardiac magnetic resonance flow measurement in the ascending aorta: breath-hold and non-breath-hold. Int J Cardiovasc Imaging 2013;29:113-20.

5 Muzzarelli S, Monney P, O'Brien K, et al. Quantification of aortic flow by phase-contrast magnetic resonance in patients with bicuspid aortic valve. Eur Heart J Cardiovasc Imaging 2014;15:77-84.

6 Śpiewak M, Kłopotowski M, Gawor M, et al. Quantification of mitral regurgitation in patients with hypertrophic cardiomyopathy using aortic and pulmonary flow data: impacts of left ventricular outflow tract obstruction and different left ventricular segmentation methods. J Cardiovasc Magn Reson 2017;19:105.

7 Chaturvedi A, Hamilton-Craig C, Cawley PJ, et al. Quantitating aortic regurgitation by cardiovascular magnetic resonance: significant variations due to slice location and breath holding. Eur Radiol 2016;26:3180-9.

8 Bertelsen L, Svendsen JH, Køber L, et al. Flow measurement at the aortic root-impact of location of through-plane phase contrast velocity mapping. J Cardiovasc Magn Reson 2016;18:55.

9 Garg P, Swift AJ, Zhong L, et al. Assessment of mitral valve regurgitation by cardiovascular magnetic resonance imaging. Nat Rev Cardiol 2020;17:298-312.
10 Myerson SG, Francis JM, Neubauer S. Direct and indirect quantification of mitral regurgitation with cardiovascular magnetic resonance, and the effect of heart rate variability. MAGMA 2010;23:243-9.

11 Uretsky S, Gillam L, Lang R, et al. Discordance between echocardiography and MRI in the assessment of mitral regurgitation severity: a prospective multicenter trial. J Am Coll Cardiol 2015;65:1078-88.

12 Uretsky S, Supariwala A, Nidadovolu P, et al. Quantification of left ventricular remodeling in response to isolated aortic or mitral regurgitation. J Cardiovasc Magn Reson 2010;12:32.

13 Sachdev V, Hannoush H, Sidenko S, et al. Are echocardiography and CMR really discordant in mitral regurgitation? JACC CardiovasC Imaging 2017;10:823-4.

14 Andersson C, Kihlberg J, Ebbers T, et al. Phase-contrast MRI volume flow - a comparison of breath held and navigator based acquisitions. BMC Med Imaging 2016;16:26.

15 Rigsby CK, Hilpipre N, McNeal GR, et al. Analysis of an automated background correction method for cardiovascular MR phase contrast imaging in children and young adults. Pediatr Radiol 2014;44:265-73.

16 van den Hout RJ, Lamb HJ, van den Aardweg JG, et al. Real-time MR imaging of aortic flow: influence of breathing on left ventricular stroke volume in chronic obstructive pulmonary disease. Radiology 2003;229:513-9.

17 Sakuma H, Kawada N, Kubo H, et al. Effect of breath holding on blood flow measurement using fast velocity encoded cine MRI. Magn Reson Med 2001;45:346-8.

18 Dahiya A, Bolen M, Grimm RA, et al. Development of a consensus document to improve multireader concordance and accuracy of aortic regurgitation severity grading by echocardiography versus cardiac magnetic resonance imaging. Am J Cardiol 2012;110:709-14.

19 Lankhaar J-W, Hofman MBM, Marcus JT, et al. Correction of phase offset errors in main pulmonary artery flow quantification. $J$ Magn Reson Imaging 2005;22:73-9.

20 O'Brien KR, Cowan BR, Jain M, et al. MRI phase contrast velocity and flow errors in turbulent stenotic jets. J Magn Reson Imaging 2008;28:210-8.

21 Bland JM, Altman DG. Statistical methods for assessing agreement between two methods of clinical measurement. Lancet 1986;1:307-10.

22 Aplin M, Kyhl K, Bjerre J, et al. Cardiac remodelling and function with primary mitral valve insufficiency studied by magnetic resonance imaging. Eur Heart $J$ Cardiovasc Imaging 2016;17:863-70.

23 Myerson SG, d'Arcy J, Christiansen JP, et al. Determination of clinical outcome in mitral regurgitation with cardiovascular magnetic resonance quantification. Circulation 2016;133:2287-96.

24 Penicka M, Vecera J, Mirica DC, et al. Prognostic implications of magnetic resonance-derived quantification in asymptomatic patients with organic mitral regurgitation: comparison with Doppler echocardiography-derived integrative approach. Circulation 2018;137:1349-60.

25 D'Errico L, Lamacie MM, Jimenez Juan L, et al. Effects of slice orientation on reproducibility of sequential assessment of right ventricular volumes and ejection fraction: short-axis vs transverse SSFP cine cardiovascular magnetic resonance. J Cardiovasc Magn Reson 2016;18:60.

26 Grothues F, Smith GC, Moon JCC, et al. Comparison of interstudy reproducibility of cardiovascular magnetic resonance with twodimensional echocardiography in normal subjects and in patients with heart failure or left ventricular hypertrophy. Am J Cardiol 2002;90:29-34.

27 Gulsin GS, Singh A, McCann GP. Cardiovascular magnetic resonance in the evaluation of heart valve disease. BMC Med Imaging 2017;17:67.

28 Kilner PJ, Gatehouse PD, Firmin DN. Flow measurement by magnetic resonance: a unique asset worth optimising. $J$ Cardiovasc Magn Reson 2007;9:723-8.

29 Lotz J, Meier C, Leppert A, et al. Cardiovascular flow measurement with phase-contrast MR imaging: basic facts and implementation. Radiographics 2002;22:651-71.

30 Chew PG, Dobson LE, Garg P, et al. CMR quantitation of change in mitral regurgitation following transcatheter aortic valve replacement (TAVR): impact on left ventricular reverse remodeling and outcome. Int J Cardiovasc Imaging 2019;35:161-70.

31 Nordmeyer S, Riesenkampff E, Messroghli D, et al. Four-dimensional velocity-encoded magnetic resonance imaging improves blood flow quantification in patients with complex accelerated flow. J Magn Reson Imaging 2013;37:208-16. 
32 Crandon S, Elbaz MSM, Westenberg JJM, et al. Clinical applications of intra-cardiac four-dimensional flow cardiovascular magnetic resonance: a systematic review. Int J Cardiol 2017;249:486-93

33 Bollache E, van Ooij P, Powell A, et al. Comparison of 4D flow and $2 \mathrm{D}$ velocity-encoded phase contrast MRI sequences for the evaluation of aortic hemodynamics. Int J Cardiovasc Imaging 2016;32:1529-41.

34 Greil G, Geva T, Maier SE, et al. Effect of acquisition parameters on the accuracy of velocity encoded cine magnetic resonance imaging blood flow measurements. J Magn Reson Imaging 2002;15:47-54. 